DOI: $10.18027 / 2224-5057-2021-11-3 s 2-05$

Цитирование: Орел Н.Ф., Артамонова Е.В., Горбунова В.А., Делекторская В.В., Емельянова Г.С., Кузьминов А.Е. и соавт. Практические рекомендации по лекарственному лечению нейроэндокринных неоплазий лёгких и тимуса. Злокачественные опухоли : Практические рекомендации RUSSCO \#3s2, 2021 (том 11). 05

\title{
ПРАКТИЧЕСКИЕ РЕКОМЕНААЦИИ ПО ЛЕКАРСТВЕННОМУ ЛЕЧЕНИЮ НЕЙРОЭНДОКРИННЫХ НЕОПЛАЗИЙ ЛЁГКИХ И ТИМУСА
}

Коллектив авторов: Орел Н.Ф., Артамонова Е.В., Горбунова В.А., Делекторская В.В., Емельянова Г.С., Кузьминов А.Е., Любимова Н.В., Маркович А.А., Орлов С.В.

Ключевые слова: нейроэндокринные опухоли, лёгкое, тимус иммунотерапия, гормонотерапия, химиотерапия, аналоги соматостатина, рецепторы соматостатина

\section{1. КЛАССИФИКАЦИЯ И ОПРЕДЕЛЕНИЕ СТАДИИ}

\section{1. По степени злокачественности}

Алгоритм выбора лечения нейроэндокринных опухолей (НЭО) лёгких и тимуса зависит от типа и распространенности опухоли и ориентирован на рекомендации ENETS 2015 г. (табл. 1). В группу G1-G2 входят высокодифференцированные НЭО лёгких и тимуса (типичный и атипичный карциноиды); в группу G3 - низкодифференцированные нейроэндокринные карциномы (крупноклеточный нейроэндокринный рак, мелкоклеточный рак). Роль индекса Кі67 в системе гистологической градации НЭО лёгких и тимуса окончательно не определена, однако, в классификацию ВО3, последнее 5-е издание которой вышло в 2021 г., включены его условные значения (наряду с митозами и некрозами) в качестве дополнительных параметров для анализа диагностических биопсий (табл. 1).

Таблица 1. Классификация нейроэндокринных опухолей лёгких и тимуса

\begin{tabular}{|l|l|l|l|l|}
\hline Гистологический тип & Некрозы & $\begin{array}{l}\text { Количество митозов } \\
\text { в 10 полях зрения (2 мм²) }\end{array}$ & $\begin{array}{l}\text { Индекс Кі-67, \% } \\
\text { (условно) }\end{array}$ & $\begin{array}{l}\text { Степень зло- } \\
\text { качественности }\end{array}$ \\
\hline $\begin{array}{l}\text { Типичный карциноид/ } \\
\text { НЭО G1 }\end{array}$ & Нет & $<2$ & До 5 & Низкая (G1) \\
\hline $\begin{array}{l}\text { Атипичный карциноид/ } \\
\text { НЭО С2 }\end{array}$ & $\begin{array}{l}\text { Нет/ } \\
\text { фокальные }\end{array}$ & $2-10$ & До 30 & $\begin{array}{l}\text { Промежуточная } \\
\text { (С2) }\end{array}$ \\
\hline $\begin{array}{l}\text { Крупноклеточный } \\
\text { нейроэндокринный рак }\end{array}$ & Обширные & $>10$ (в среднем -70) & $30-100$ & Высокая (G3) \\
\hline
\end{tabular}




\begin{tabular}{|l|l|l|l|l|}
\hline Гистологический тип & Некрозы & $\begin{array}{l}\text { Количество митозов } \\
\text { в 10 полях зрения (2 мм²) }{ }^{1}\end{array}$ & $\begin{array}{l}\text { Индекс Кі-67, \% } \\
\text { (условно) }\end{array}$ & $\begin{array}{l}\text { Степень зло- } \\
\text { качественности }\end{array}$ \\
\hline Мелкоклеточный рак & Обширные & $>10$ (в среднем -80) & $30-100$ & Высокая (G3) \\
\hline
\end{tabular}

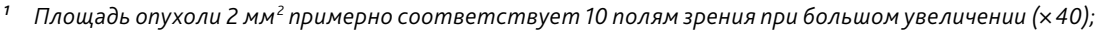
для определения данного показателя оцениваются не менее 50 полей в областях наибольшей митотической плотности.

WHO Classification of Tumours Editorial Board. Thoracic tumours. Lyon (France): International Agency for Research on Cancer; 2021. (WHO classification of tumours series, 5th ed.; vol. 5).

В 2018 г. опубликованы предложения ВОЗ по созданию универсальной классификации для нейроэндокринных неоплазий (НЭН) различной локализации, включая легкое и тимус (табл. 2) [Rindi G, 2018]. Эти предложения четко разделяют НЭН на два больших семейства: высокодифференцированные НЭН/нейроэндокринные опухоли - НЭО и низкодифференцированные НЭН, нейроэндокринные карциномы/рак - НЭР. Следует подчеркнуть, что все НЭО имеют три степени злокачественности (G1, G2 и G3). НЭО G3 являются высокодифференцированными новообразованиями с повышенной пролиферативной активностью (с индексом Ki -67 > 20\%) и нередко развиваются в результате опухолевой прогрессии [Rindi G, 2020]. НЭР всегда относится к новообразованиям высокой степени злокачественности и не требует дополнительного цифрового уточнения G. В морфологическом заключении рекомендуется использовать новую номенклатуру в скобках (NEN-WHO 2018), наряду с традиционной терминологией, которая утверждена действующей классификацией ВОЗ.

Таблица 2. Универсальная классификация нейроэндокринных неоплазий, независимо от локализации (NEN-WHO 2018 г.)

\begin{tabular}{|c|c|c|c|c|c|}
\hline Локализация & Категория & Семейство & Тип & $\begin{array}{l}\text { Степень } \\
\text { злокаче- } \\
\text { ственности }\end{array}$ & $\begin{array}{l}\text { Действующая } \\
\text { терминология }\end{array}$ \\
\hline \multirow[t]{4}{*}{ Легкое/Тимус } & \multirow{4}{*}{$\begin{array}{l}\text { Нейроэндо- } \\
\text { кринная } \\
\text { неоплазия }\end{array}$} & \multirow{3}{*}{$\begin{array}{l}\text { Нейро- } \\
\text { эндокринная } \\
\text { опухоль } \\
\text { (НЭО) }\end{array}$} & \multirow[t]{3}{*}{$\begin{array}{l}\text { НЭО легкого НЭО } \\
\text { тимуса }\end{array}$} & G1 & $\begin{array}{l}\text { Типичный } \\
\text { карциноид }\end{array}$ \\
\hline & & & & G2 & \multirow{2}{*}{$\begin{array}{l}\text { Атипичный } \\
\text { карциноид }\end{array}$} \\
\hline & & & & G3 & \\
\hline & & $\begin{array}{l}\text { Нейро- } \\
\text { эндокринная } \\
\text { карци- } \\
\text { нома/рак } \\
\text { (НЭР) }\end{array}$ & $\begin{array}{l}\text { НЭР легкого, } \\
\text { мелкоклеточный тип } \\
\text { (МРЛ }{ }^{1} \\
\text { НЭР легкого, крупно- } \\
\text { клеточный тип } \\
\text { НЭР тимуса, } \\
\text { мелкоклеточный тип } \\
\text { (МРЛ) } \\
\text { НЭР тимуса, крупно- } \\
\text { клеточный тип }\end{array}$ & & $\begin{array}{l}\text { Мелкоклеточный } \\
\text { рак } \\
\text { Крупноклеточный } \\
\text { нейроэндокрин- } \\
\text { ный рак } \\
\text { Мелкоклеточный } \\
\text { рак } \\
\text { Крупноклеточный } \\
\text { нейроэндокрин- } \\
\text { ный рак }\end{array}$ \\
\hline
\end{tabular}


1 Исключение составляет термин «мелкоклеточный рак легкого - МРЛ», который предложено не менять, учитывая его широкое практическое использование.

Rindi G, Klimstra DS. et al. A common classification framework for neuroendocrine neoplasms: an International Agency for Research on Cancer (IARC) and World Health Organization (WHO) expert consensus proposal. Modern Pathology. 2018; 31 (12): 1770-1786

Rindi G, Inzani F. Neuroendocrine neoplasm update: toward universal nomenclature Endocrine-Related Cancer. 2020; $27, R 211-R 218$

\section{2. Классификация по системе TNM}

Опухоли должны быть стадированы в соответствии с TNM-классификацией органа, в котором возникли. В настоящее время в России используется 8-е издание классификации TNM.

\section{2. ДИАГНОСТИКА}

Диагноз НЭО лёгких и тимуса основывается на данных осмотра, результатах инструментальных методов обследования и патоморфологическом заключении. Первичное обследование должно быть проведено до всех видов лечебных воздействий и включает в себя:

- сбор анамнеза и осмотр; осмотр включает пальпацию лимфоузлов регионарных зон, а также выявление симптомов, подозрительных в отношении отдаленных метастазов и наличия синдромов;

- общий анализ крови с подсчетом лейкоцитарной формулы и количества тромбоцитов;

- биохимический анализ крови с определением показателей функции печени, почек, кальция, глюкозы, электролитов (натрий);

- УзИ органов брюшной полости, забрюшинного пространства, почек, надпочечников, периферических лимфоузлов (шейно-надключичных, подмышечных) и малого тазапо показаниям;

- R-графию органов грудной клетки;

- КТ/МРТ органов брюшной полости и малого таза с контрастированием - по показаниям;

- КТ органов грудной клетки;

- КТ-ангиографию органов грудной клетки - по показаниям для дифференциальной диагностики;

- радиоизотопное исследование скелета+R-графию и/или КT/MPT зон накопления РФП - по показаниям;

- бронхоскопию;

- биопсию опухоли с патоморфологическим исследованием опухолевой ткани и определением митотического индекса;

- ИГХ определение в опухолевой ткани хромогранина А, синаптофизина, Кі67, рецепторов соматостатина 2A и 5 типа - по показаниям; В сложных случаях - дополнительное ИГХ исследование цитокератина 18, CD56, хромогранина В и INSM1. 
- определение биохимических маркеров: кровь - хромогранин А, серотонин, НСЕ (при G2-G3); АКТГ, кортизол, гистамин - по показаниям; моча - 5-ГИУК; NTproBNP в крови - при наличии карциноидного синдрома ежегодно;

- сцинтиграфия с $\ln ^{111}$ (октреоскан) - по показаниям (для G1, G2);

- КТ, МРТ головного мозга - по показаниям;

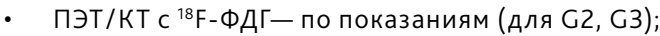

- ПЭT/KT c ${ }^{68} \mathrm{Ga}$-DOTA-TOC, DOTA-NOC, DOTA-TATE (DOTA конъюгаты рецепторов соматостатина) - по показаниям (для $\mathrm{G} 1, \mathrm{G} 2)$;

- сцинтиграфия с пентетреодитом и/или 99mтс-Тектротидом по показаниям (для G1, G2);

- ЭхоКГ - 1 раз в 6 мес. при наличии карциноидного синдрома;

- консультация медицинского генетика - по показаниям (для больных с синдромом МЭН1).

\section{3. ЛЕЧЕНИЕ}

\section{1. Лекарственное}

\subsection{1. Гормонотерапия (аналоги соматостатина)}

\subsubsection{1. Гормонотерапия при HЭO G1/G2}

Аналоги соматостатина пролонгированного действия (октреотид и ланреотид) как самостоятельное лечение применяются при высокодифференцированных НЭО лёгких: октреотид пролонгированный (депо, лонг) 30 мг в/м 1 раз в 28 дней, ланреотид 120 мг п/к 1 раз в 28 дней в качестве 1-й линии терапии для контроля карциноидного синдрома и в качестве антипролиферативного средства. Возможны комбинации октреотида-депо (30 мг 1 раз в 28 дней в/м) с ИФН- $\alpha$, цитостатиками, таргетными препаратами в случае быстрого прогрессирования распространенной болезни. В соответствии с инструкцией по использованию пролонгированных форм октреотида лечение начинается с октреотида короткого действия. Октреотид короткого действия применяется п/к в дозе 100 мкг 3 раза в сутки в течение 2 недель перед назначением пролонгированных форм, затем при удовлетворительной переносимости рекомендуется пролонгированный октреотид длительного действия в дозе 30 мг в/м. В случае прогрессирования на первоначальной дозе дозу пролонгированного октреотида следует повысить до 40-60 мг 1 раз в 28 дней или следует уменьшить интервал между введением аналогов соматостатина до 1 раза в 21-14 дней (табл. 3). Для ланреотида рекомендуемая доза составляет 120 мг п/к каждые 4 недели, при прогрессировании возможно уменьшение интервала между введениями до 3-2 недель или эскалация дозы до 180 мг при наличии дозировки 60 мг или 90 мг. Данное лечение проводится до прогрессирования или непереносимой токсичности. При прогрессировании заболевания дозу аналогов соматостатина необходимо увеличить либо сократить интервалы между введениями. В случае использования комбинированного режима лечения второй компонент комбинированной терапии (таргетный препарат, цитостатик, ИФН- $\alpha$ ) следует отменить и заменить на другое лечение. После завершения курса комбинированного 
лекарственного лечения, а также при монотерапии лечение аналогами соматостатина продолжают длительно. При непереносимости какого-либо препарата или прогрессировании опухолевого процесса один препарат может быть заменен на другой (октреотид на ланреотид и наоборот).

Таблица 3. Рекомендуемые режимы применения аналогов соматостатина при нейроэндокринных опухолях лёгких и тимуса

\begin{tabular}{|l|l|}
\hline Препарат & Режим применения \\
\hline Октреотид & $\begin{array}{l}100 \text { мкг } 3 \text { раза/сут. п/к в течение } 2 \text { нед., далее-переход } \\
\text { на пролонгированные формы }\end{array}$ \\
\hline Октреотид пролонгированный (депо, лонг) & 30 мг в/м 1 раз в 28 дней \\
\hline Ланреотид пролонгированного действия & 120 мг п/к 1 раз в 28 дней \\
\hline
\end{tabular}

\subsubsection{2. Гормонотерапия при НЭР}

Аналоги соматостатина пролонгированного действия при нейроэндокринных карциномах используются для контроля симптомов или при наличии экспрессии рецепторов соматостатина в опухоли применяются в комбинации с цитостатиками в дозе 20 мг 1 раз в 28 дней в/м для октреотида и 120 мг 1 раз в 28 дней п/к для ланреотида.

\subsection{2. Таргетная терапия}

\subsubsection{1. Таргетная терапия при $\mathrm{H} Э \mathrm{O} \mathrm{G} 1 / \mathrm{G} 2$}

Эверолимус является единственным таргетным препаратом, зарегистрированным для лечения высокодифференцированных НЭО лёгких. Препарат может использоваться в качестве антипролиферативной терапии во II-III линиях лечения после прогрессирования на фоне терапии аналогами соматостатина при диссеминированном процессе в комбинации с аналогами соматостатина, а также в I линии лечения при отсутствии рецепторов к соматостатину или невозможности ХТ в монотерапии. Эверолимус используется в дозе 10 мг/сутки ежедневно внутрь до прогрессирования или непереносимой токсичности; при развитии симптомов токсичности может использоваться доза 5 мг/сут. (табл. 4).

Таблица 4. Рекомендуемый режим использования эверолимуса при НЭО лёгких и тимуса

\begin{tabular}{|l|l|}
\hline Препарат & Режим применения \\
\hline Эверолимус & 10 мг/сут. внутрь ежедневно длительно \\
\hline
\end{tabular}

\subsubsection{2. Таргетная терапия при НЭР}

Применение таргетной терапии при низкодифференцированных НЭО не рекомендовано. 


\subsection{3. Иммунотерапия}

\subsubsection{1. Иммунотерапия при $\mathrm{H} Э \mathrm{O} \mathrm{G} 1 / \mathrm{G2}$}

ИФН- $\alpha$ является терапией ІІ линии при высокодифференцированных НЭО. ИФН- $\alpha$ рекомендуется использовать как дополнение к терапии аналогами соматостатина при недостаточном контроле симптомов функционирующих опухолей. Кроме того, ИФН- $\alpha$ может использоваться в качестве терапии I линии при отсутствии экспрессии рецепторов соматостатина. Рекомендуемая доза ИФН- $\alpha$ составляет 3 млн. МЕ п/к 3 раза в нед. (табл. 5). При непереносимости стандартного режима введения может быть рекомендован пегилированный ИФН- $\alpha$ (50-180 мкг/нед. п/к).

Таблица 5. Рекомендуемый режим использования ИФН- $\alpha$ при НЭО G1/G2 лёгких и тимуса

\begin{tabular}{|l|l|}
\hline Препарат & Режим применения \\
\hline ИФН- $\alpha$ & 3 млн. МЕ п/к 3 раза в нед. длительно \\
\hline
\end{tabular}

\subsubsection{2. Иммунотерапия в качестве III и последующих линий при НЭР}

Рекомендована только при МРЛ (см. раздел лекарственное лечение МРЛ)

Таблица 6. Рекомендуемые режимы иммунотерапии

\begin{tabular}{|l|l|}
\hline Ниволумаб & $\mathbf{3}$ мг/кг в/в каждые 2 нед. \\
\hline Пембролизумаб & $\begin{array}{l}2 \text { мг/кг каждые } 3 \text { нед. в/в (суммарная разовая доза } \\
\text { не должна превышать 200 мг) }\end{array}$ \\
\hline
\end{tabular}

\subsection{4. Химиотерапия}

Применение XТ при НЭО лёгких и тимуса G1 в I линии терапии не рекомендуется, за исключением случаев отсутствия эффекта от предшествующего лечения (ГТ, иммунотерапии, таргетной терапии) или при быстром прогрессировании. При НЭО G2 применение XT ( \pm аналоги соматостатина) в I линии рекомендуется при значительной массе опухоли и наличии карциноидного синдрома (табл. 7). При НЭР основным способом лечения является XT, предпочтительными режимами I линии являются комбинации ЕР или ЕС (табл. 8). Кроме того, в 2020 г. оригинальный отечественный препарат Араноза (арабинопиранозилметил нитрозомочевина) был зарегистрирован по новому показанию: монотерапия нейроэндокринных опухолей различной локализации. Араноза (арабинопиранозилметил нитрозомочевина) также может использоваться в сочетании с аналогами соматостатина. 
Таблица 7. Рекомендуемые режимы химиотерапии при НЭО G2 лёгких и тимуса

\begin{tabular}{|c|c|}
\hline Режим & Схема \\
\hline $\begin{array}{l}\text { Араноза (арабинопиранозилметил } \\
\text { нитрозомочевина) }\end{array}$ & 500-650 мг/м² в/в струйно 1-3 дни 1, каждые 3-4 недели \\
\hline XELOX & $\begin{array}{l}\text { Оксалиплатин } 130 \text { мг/м² в/в в 1-й день + капецитабин } \\
2000 \text { мг/м² внутрь в 1-14-й дни, каждые } 3 \text { недели }\end{array}$ \\
\hline Капецитабин & Капецитабин 2500 мг/м²/сут. внутрь в 1-14-й дни, каждые 3 недели \\
\hline $\begin{array}{l}\text { Капецитабин } \\
\text { (метрономный режим) }\end{array}$ & $\begin{array}{l}\text { Капецитабин } 500 \text { мг внутрь 2-3 раза в сутки ежедневно длительно, } \\
\text { до прогрессирования или непереносимой токсичности }\end{array}$ \\
\hline TemCap & $\begin{array}{l}\text { Темозоломид } 150 \text { мг/м²/сут. внутрь в 10-14-й дни + капецитабин } \\
2000 \text { мг/м²/сут. внутрь в 1-14-й дни, каждые } 4 \text { недели }\end{array}$ \\
\hline $\mathrm{TI}$ & $\begin{array}{l}\text { Темозоломид 150-200 мг/м²/сут. внутрь в 1-5-й дни + иринотекан } \\
250 \text { мг/м² в/в в 6-й день, каждые } 4 \text { недели }\end{array}$ \\
\hline Темозоломид & Темозоломид 150-200 мг/м²/сут. внутрь в 1-5-й дни, каждые 4 недели \\
\hline $\begin{array}{l}\text { Темозоломид } \\
\text { (метрономный режим) }\end{array}$ & $\begin{array}{l}\text { Темозоломид } 75 \text { мг/м²/сут. внутрь, ежедневно длительно, до про- } \\
\text { грессирования или непереносимой токсичности }\end{array}$ \\
\hline FOLFOX & $\begin{array}{l}\text { Оксалиплатин } 85 \text { мг/м² в/в в 1-й день } \\
\text { Кальция фолинат } 400 \text { мг/м² в/в в 1-й день } \\
\text { 5-фторурацил } 400 \text { мг/м² в/в в 1-й день } \\
\text { 5-фторурацил } 2400 \text { мг/м² в/в } 46 \text { часов, } \\
\text { каждые } 2 \text { недели }\end{array}$ \\
\hline FOLFIRI & 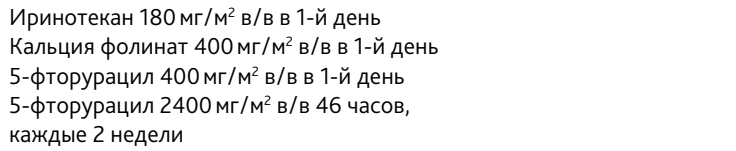 \\
\hline GEMOX & 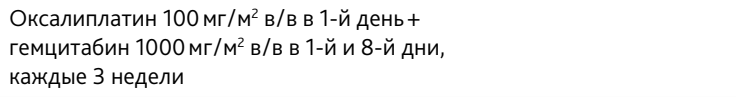 \\
\hline MGEMOX & $\begin{array}{l}\text { Оксалиплатин } 85 \text { мг/м² в/в в 1-й день + } \\
\text { гемцитабин } 1000 \text { мг/м² в/в кап 1-й день, } \\
\text { каждые } 2 \text { недели }\end{array}$ \\
\hline Паклитаксел+цисплатин & $\begin{array}{l}\text { Паклитаксел } 175 \text { мг/м² в/в в 1-й день + } \\
\text { цисплатин } 75 \text { мг/м² в/в кап в 1-й день, } \\
\text { каждые } 3 \text { недели }\end{array}$ \\
\hline Доцетаксел+цисплатин & 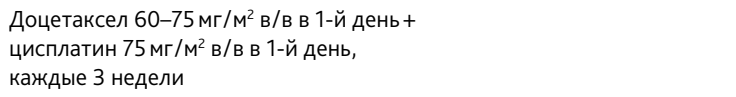 \\
\hline Паклитаксел+карбоплатин & $\begin{array}{l}\text { Паклитаксел } 175 \text { мг/м² в/в в 1-й день + } \\
\text { карбоплатин AUC 5-6 в/в в 1-й день, } \\
\text { каждые } 3 \text { недели }\end{array}$ \\
\hline Доцетаксел+карбоплатин & $\begin{array}{l}\text { Доцетаксел 60-75 мг/м² в/в в 1-й день + } \\
\text { карбоплатин AUC 5-6 в/в в 1-й день, } \\
\text { каждые } 3 \text { недели }\end{array}$ \\
\hline
\end{tabular}




\begin{tabular}{|l|l|}
\hline Режим & Схема \\
\hline Бевацизума6+XT & $\begin{array}{l}\text { Бевацизума6²5-7,5 мг/кг в/в в комбинации с XT, } \\
\text { каждые 2-3 недели }\end{array}$ \\
\hline
\end{tabular}

1 Не более 3,0 г препаратана курс

2 Для бевацизумаба не зарегистрировано показание к применению при НЭО. В клинических исследованиях применялся в комбинациях с капецитабином и темозоломидом.

Таблица 8. Рекомендуемые режимы химиотерапии при НЭР лёгких и тимуса

\begin{tabular}{|c|c|}
\hline \multicolumn{2}{|l|}{ Режимы I линии } \\
\hline EP & $\begin{array}{l}\text { Этопозид } 100 \text { мг/м² в/в в 1-3-й дни + цисплатин } 75 \text { мг/м² в/в в 1-й день } 1 \text { раз в } 3 \text { нед., } \\
6 \text { циклов }\end{array}$ \\
\hline $\mathrm{EC}$ & $\begin{array}{l}\text { Этопозид } 100 \text { мг/м² в/в в 1-3-й дни + карбоплатин AUC } 5 \text { в/в в 1-й день } 1 \text { раз в } 3 \text { нед., } \\
6 \text { циклов }\end{array}$ \\
\hline IP & $\begin{array}{l}\text { Иринотекан } 65 \text { мг/м² в/в в 1-й и 8-й дни + цисплатин } 75 \text { мг/м² в/в в 1-й день, каждые } \\
3 \text { нед., } 6 \text { циклов }\end{array}$ \\
\hline IC & $\begin{array}{l}\text { Иринотекан } 65 \text { мг/м² в/в в 1-й и 8-й дни + карбоплатин AUC } 5 \text { в/в в 1-й день, каждые } \\
3 \text { нед., } 6 \text { циклов }\end{array}$ \\
\hline \multicolumn{2}{|l|}{ Режимы II линии } \\
\hline CAV & 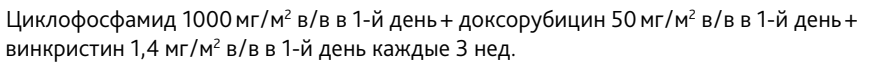 \\
\hline $\begin{array}{l}\text { Топотекан } \\
\text { (монотерапия) }\end{array}$ & 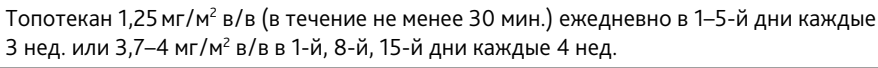 \\
\hline $\begin{array}{l}\text { Паклитак- } \\
\text { сел+карбоплатин }\end{array}$ & $\begin{array}{l}\text { Паклитаксел } 175 \text { мг/м² в/в в 1-й день + карбоплатин AUC } 5 \text { в/в в 1-й день каждые } \\
\text { 3-4 нед. }\end{array}$ \\
\hline $\begin{array}{l}\text { Доцетаксел+цис- } \\
\text { платин }\end{array}$ & Доцетаксел 75 мг/м² в/в в 1-й день + цисплатин 75 мг/м² в/в в 1-й день каждые 3 нед. \\
\hline $\begin{array}{l}\text { Гемцитабин +цис- } \\
\text { платин }\end{array}$ & $\begin{array}{l}\text { Гемцитабин } 1000 \text { мг/м² в/в в1-й, 8-й день + цисплатин } 70 \text { мг/м² в/в в 1-й день каждые } \\
3 \text { нед. }\end{array}$ \\
\hline
\end{tabular}

\section{2. Хирургическое лечение}

При операбельном процессе рекомендуется хирургическое лечение. Однозначных рекомендаций относительно нео-и адъювантного лечения при HEO G1-2 не существует. При G1 неоадъювантное лечение не проводится, при G2, G3 возможно у отдельных больных. Факторами риска являются метастазы в лимфатических узлах, а также повышение биохимических маркеров. Могут обсуждаться аналоги соматостатина, интерфероны, комбинации XT, не содержащие цисплатин, в зависимости от индекса Кі67. Решение в этих случаях должно приниматься на мультидисциплинарном консилиуме. При неоперабельных HЭO G1 и G2 хирургический метод лечения используется для уменьшения опухолевой массы (циторедуктивные операции), что особенно актуально при гормонопродуцирующих опухолях и может использоваться последовательно либо совместно с лекарственной 
терапией. Другими циторедуктивными методами являются химиоэмболизация, эмболизация и РЧА метастазов в печени. У большинства больных удается достичь значительного уменьшения проявлений карциноидного синдрома.

\section{3. Лучевое лечение}

Дистанционная ЛТ проводится с паллиативной (обезболивающей) целью. При локализованных неоперабельных НЭО G2-G3 ЛТ используется в сочетании с XT. Сочетанная ХЛТ терапия описана в соответствующем разделе (местно-распространенный мелкоклеточный рак лёгкого).

\section{4. НАБЛЮДЕНИЕ}

Задачей наблюдения является раннее выявление прогрессирования заболевания с целью своевременного начала лекарственной терапии или выполнения хирургического лечения при операбельных опухолях (табл. 9).

\section{1. Алгоритм наблюдения за больными после операции}

- 1-й год - 1 раз в 3 мес.

- 2-й год - 1 раз 3 мес.

- 3-5-й годы-1 раз в 6 мес.

- После 5 лет - 1 раз в год

\section{2. Объем обследования на этапе наблюдения и во время лечения}

- Сбор анамнеза и осмотр; осмотр включает пальпацию лимфоузлов регионарных зон, а также выявление симптомов, подозрительных в отношении отдаленных метастазов и наличия синдромов;

- общий анализ крови с подсчетом лейкоцитарной формулы и количества тромбоцитов;

- биохимический анализ крови с определением показателей функции печени, почек, кальция, глюкозы, электролитов (натрий);

- УзИ органов брюшной полости и малого таза, периферических лимфоузлов;

- R-графия органов грудной клетки;

- КТ/МРТ органов брюшной полости и малого таза с контрастированием - по показаниям;

- КТ органов грудной клетки;

- бронхоскопия - по показаниям;

- радиоизотопное исследование скелета + рентгенография и/или КТ/МРТ зон накопления РФП - по показаниям; 
- определение биохимических маркеров: кровь - хромогранин A, серотонин, НCE (G2-G3); АКТГ, кортизол - по показаниям; NTproBNP - при наличии карциноидного синдрома ежегодно, при карциноидной болезни сердца - каждые 6 мес., моча - 5 -ГИУК;

- ЭКГ;

- ЭхоКГ - 1 раз в 6 мес., при наличии карциноидного синдрома;

- сцинтиграфия с $\ln ^{111}$ (октреоскан) - по показаниям (для G1, G2);

- КТ, МРТ головного мозга - по показаниям.

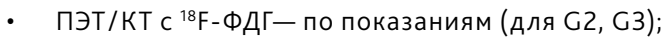

- $\quad$ ПЭT/KT c ${ }^{68} \mathrm{Ga}$-DOTA-TOC, DOTA-NOC, DOTA-TATE (DOTA конъюгаты рецепторов соматостатина) - по показаниям (для G1, G2);

- сцинтиграфия с пентетреодитом и/или ${ }^{99 m T c-Т е к т р о т и д о м ~ п о ~ п о к а з а н и я м ~(д л я ~ G 1, ~ G 2) . ~}$ Алгоритм лечебно-диагностических мероприятий при НЭО лёгких и тимуса представлен на рис. 1, 2, 3 и 4. 


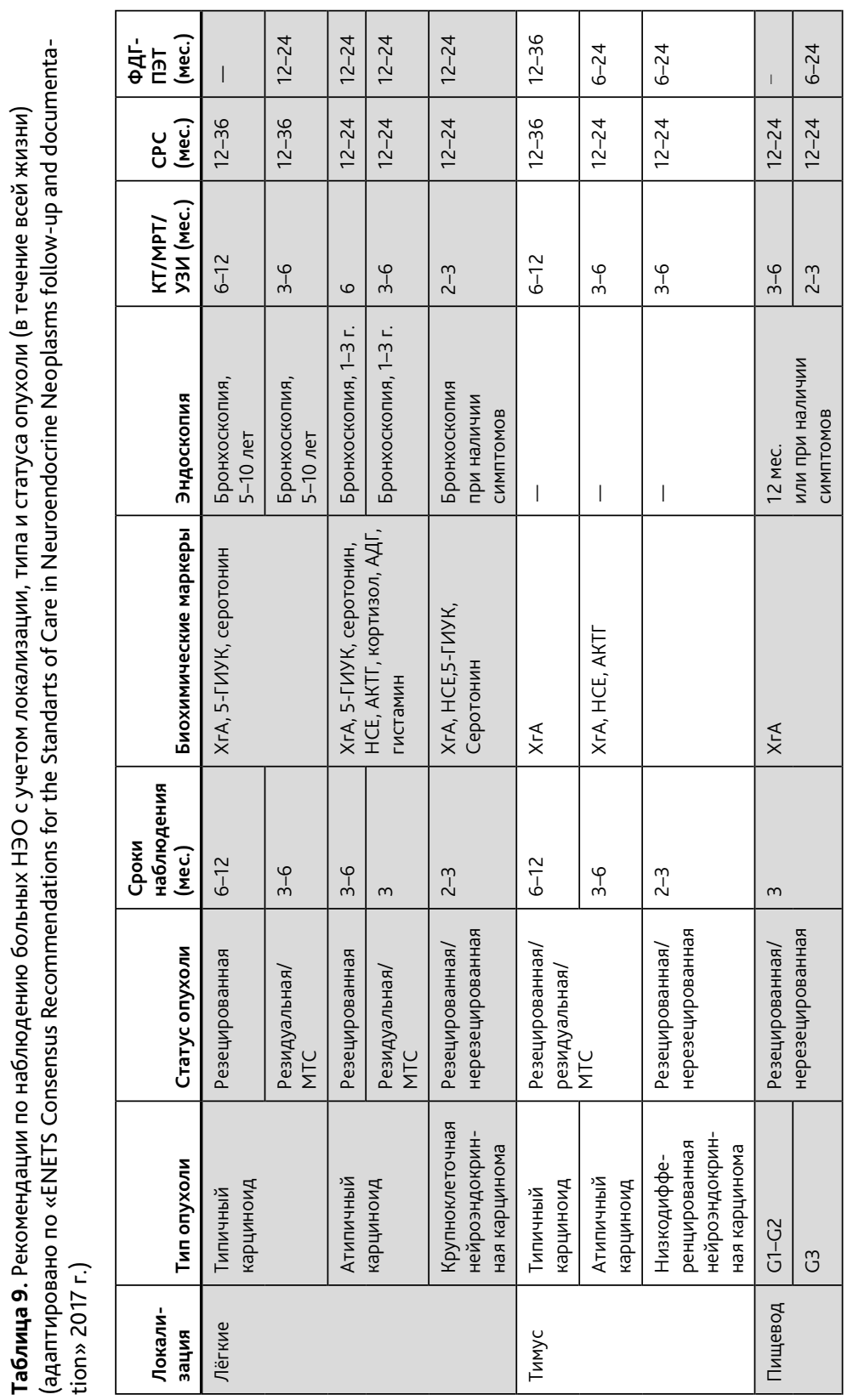




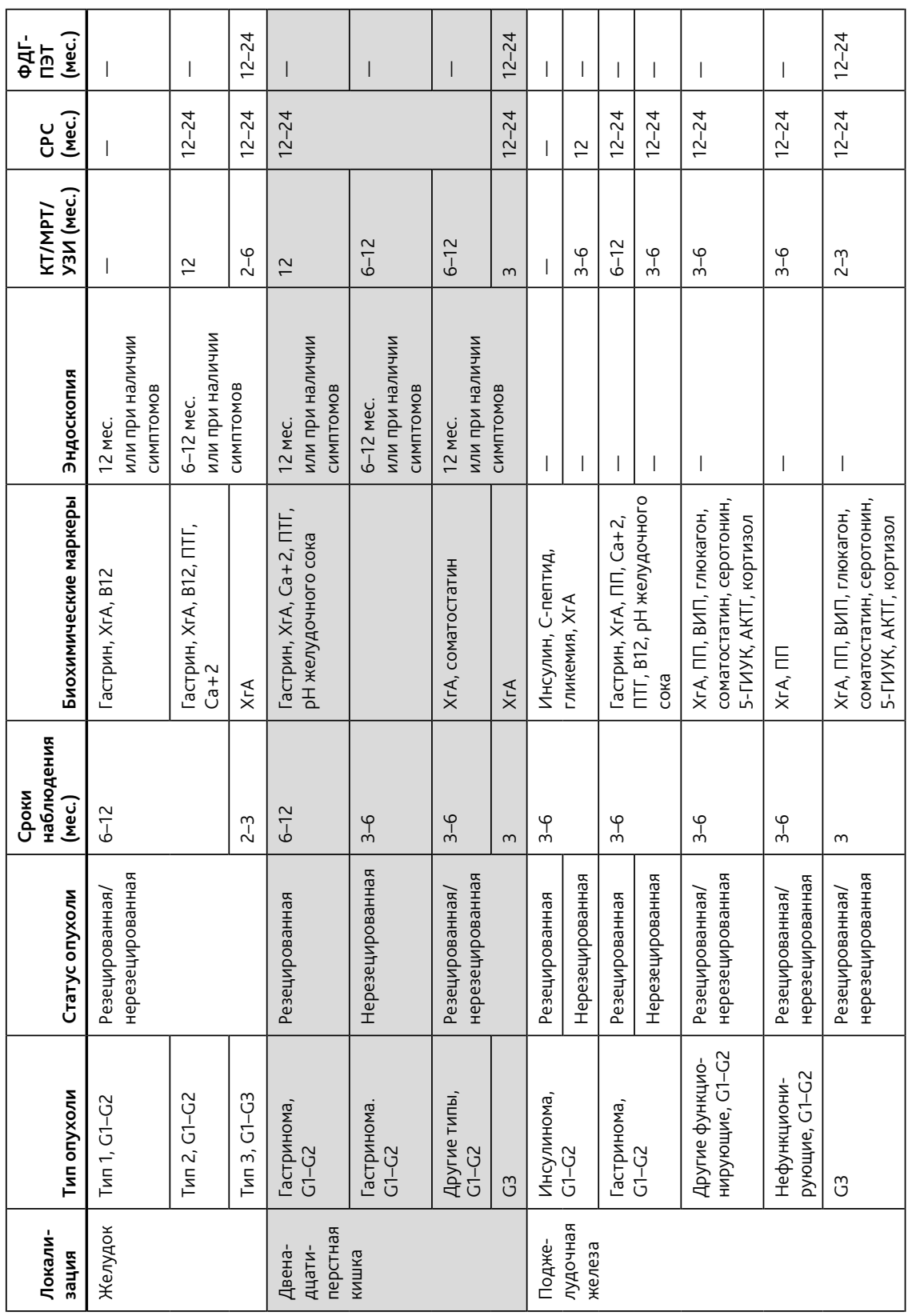




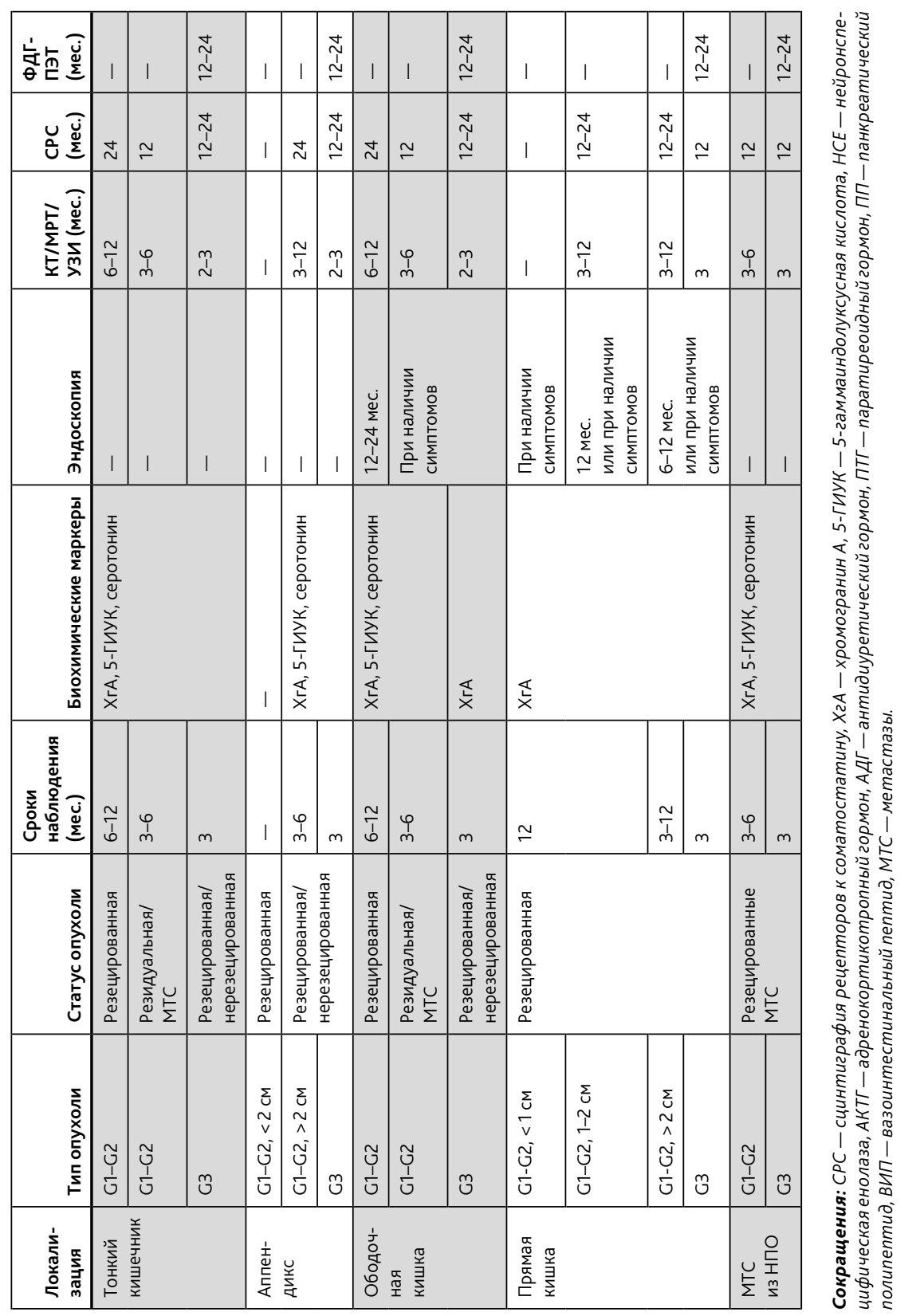



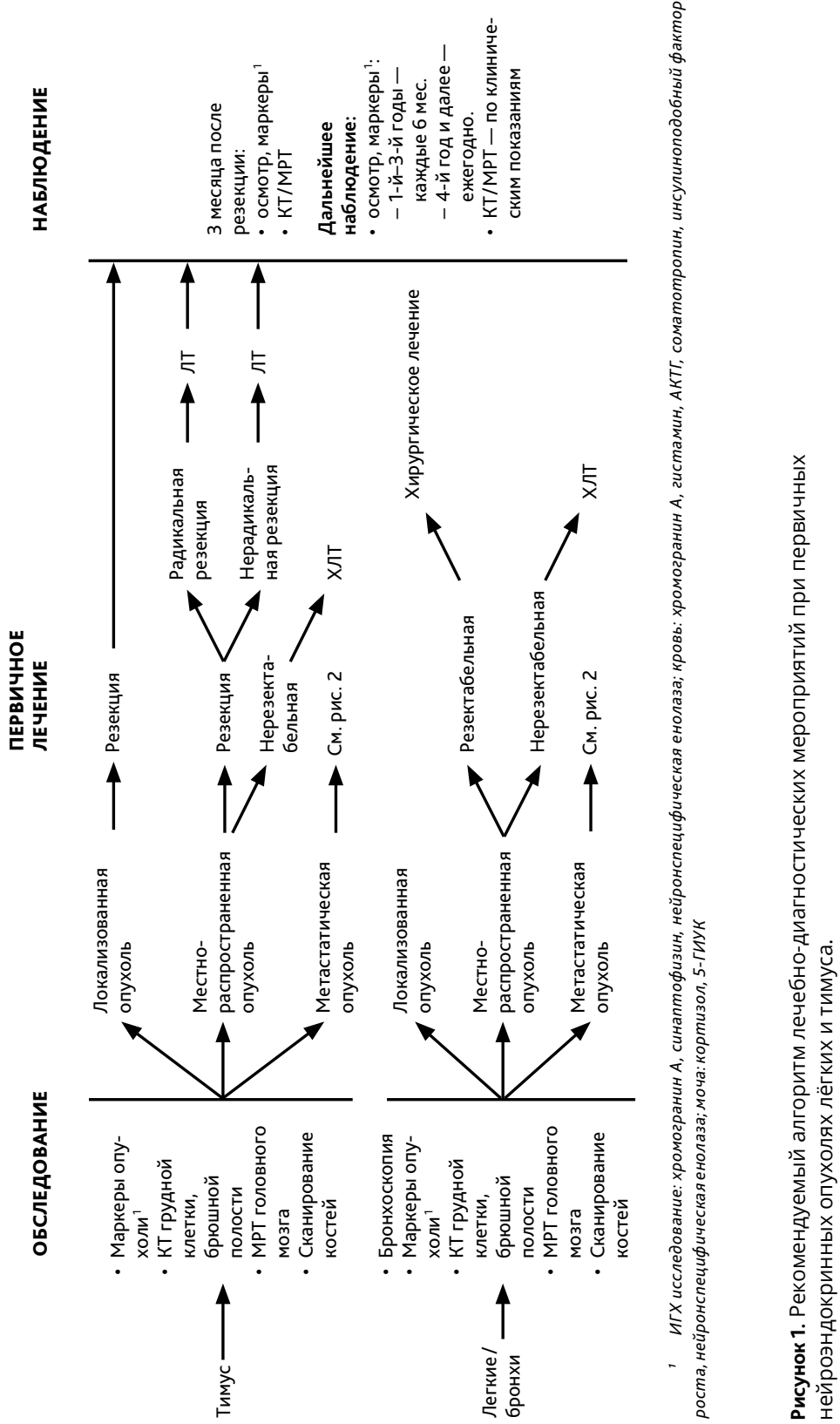

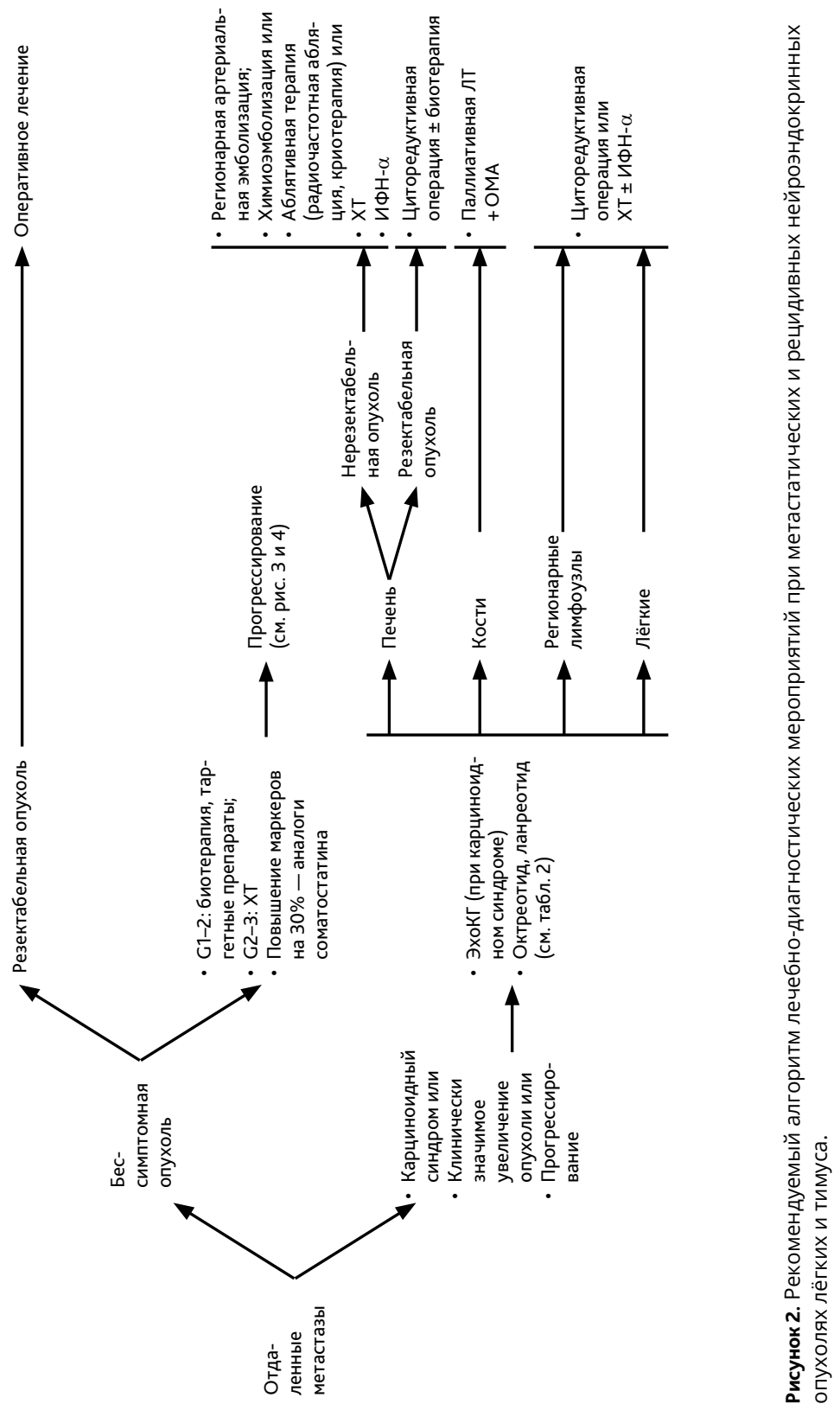


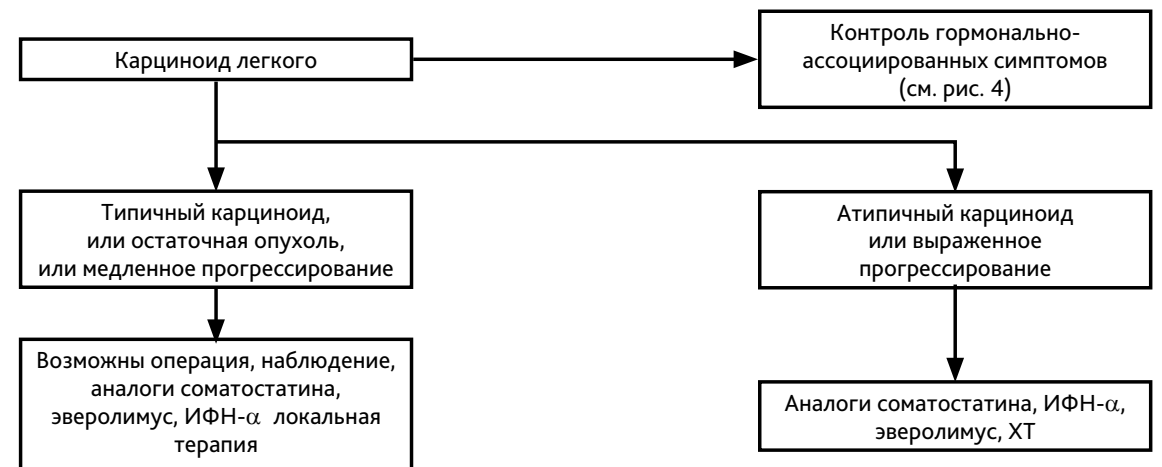

Рисунок 3. Рекомендуемый алгоритм лечения типичного и атипичного карциноидов лёгкого.

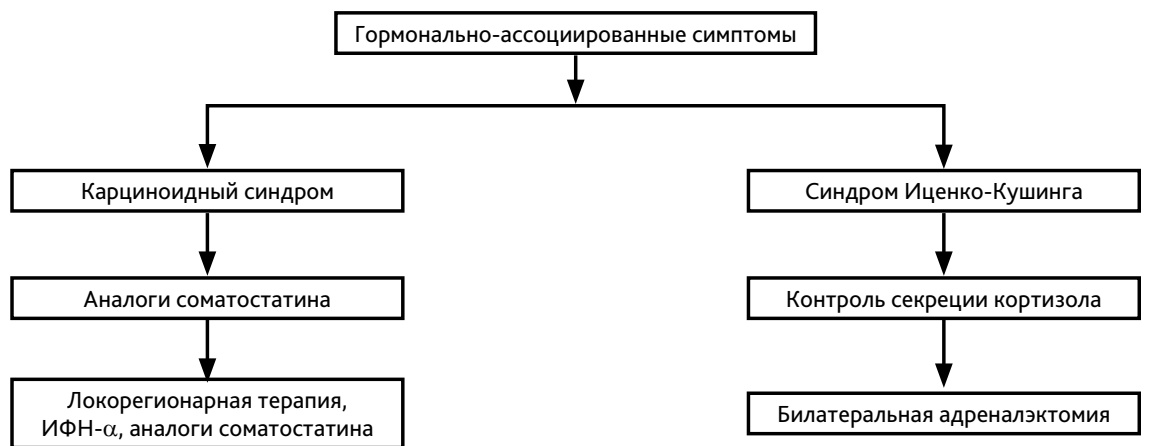

Рисунок 4. Рекомендуемый алгоритм контроля симптомов при типичном и атипичном карциноидах лёгкого 